Case Report

\title{
Rabies in a Vaccinated 9-Month-Old German Shepherd Dog, Akure, 2010: A Case Report
}

\author{
A. M. Qasim, ${ }^{1}$ A. A. Obadua, ${ }^{2}$ P. A. Okewole, ${ }^{3}$ I. S. Tekki, ${ }^{3}$ and O. S. Omoleye ${ }^{2}$ \\ ${ }^{1}$ National Veterinary Research Institute, Vom PMB 01, Nigeria \\ ${ }^{2}$ State Veterinary Hospital, Akure, Ondo State, Nigeria \\ ${ }^{3}$ Central Diagnostic Department, National Veterinary Research Institute, Vom PMB 01, Nigeria \\ Correspondence should be addressed to A. M. Qasim; abdullahifirm@yahoo.com
}

Received 2 February 2013; Accepted 21 March 2013

Academic Editors: F. Mutinelli and D. M. Wong

Copyright (C) 2013 A. M. Qasim et al. This is an open access article distributed under the Creative Commons Attribution License, which permits unrestricted use, distribution, and reproduction in any medium, provided the original work is properly cited.

\begin{abstract}
After the onset of symptoms, the clinical course of rabies is almost invariably fatal. Rabies has traditionally been associated with dogs more than any other animal, and in parts of the world where domestic animal control and vaccination programs are limited, dogs remain the most important reservoir of the disease. We report a case of canine rabies in a vaccinated 9-month-old German shepherd female dog. The presenting clinical sign was jaw muscle paralysis with a hanging bronze color like tongue without salivation. Following encephalectomy, a rabies positive diagnosis was confirmed by fluorescent antibody technique at the Rabies Laboratory, National Veterinary Research Institute, Vom. The epidemiology of the rabies case is not understood. This case is of public health significance because of the at-risk population including animal health care service provider and animals. The following were recommended, (a) a reinvigorated control measure that includes the awareness program on prevention, responsible dog ownership with dog registration at veterinary hospitals, and registered veterinary clinics by the government and (b) a yearly rabies vaccination campaign that must be improved through the veterinary public health and other health departments collaborating.
\end{abstract}

\section{Introduction}

The relationship between man and dogs spans several centuries [1]. Rabies is a disease of great public health importance because the case fatality rate is almost $100 \%$ [2]. The etiologic agents of rabies are members of the Rhabdoviridae, genus Lyssavirus, with seven recognized genotypes [2,3].

Canine rabies is endemic in Nigeria. In a study in the National Veterinary Research Institute (NVRI), Vom, 46.9\% (153/326) laboratory confirmed cases were recorded during January 2006-December 2007; 24.8\% (38/153) were puppies aged 1-6 month; $70 \%$ (107/153) were dogs $\geq 7$-month age [4].

Delays in diagnosis greatly increase the number of contacts that require postexposure prophylaxis. In the USA, one case resulted in 209 postexposure treatments (PET). The early diagnosis of rabies is also essential to eliminate the expense and discomfort of unnecessary diagnostic tests and inappropriate therapy [5].

Sustained vaccination programs are essential for adequacy of coverage and immunocompetence of the reservoir population. Most importantly, because the exposure risks are quite high in urban settings [6] particularly, this case, being vaccinated. This study is aimed at determining rabies in a vaccinated dog with a view to improve this rabies control measure.

\section{Case History}

A 9-month-old German shepherd dog was presented to the Veterinary Hospital, Akure, Ondo State, on June 26, 2010. The chief complaint was that the dog refused food and water for 2 days and a changed behavior was noticed. It was further revealed that the dog was vaccinated using the National Veterinary Research Institute, Vom, vaccine on February 1, 2010. The dog lived in an unfenced apartment. There were no reported human and animal contacts. Clinical examination revealed a protruded tongue, that is, bronze like, paralyzed jaw muscles, restlessness and occasional howling, and a rectal temperature of $39.4^{\circ} \mathrm{C}$. Hydrophobia with dilated 
pupils was observed. There was an absence of buccal swelling or foreign body lodgment on buccal cavity examination. The dog was quarantined subsequently for 12 days and managed. Progressively, the observed signs deteriorated by the days, with recumbency and mortality occurring on July 4, 2010. Then, the head was severed from the neck, prepared in cold packs, and sent by road to the Rabies Laboratory, National Veterinary Research Institute (NVRI), Vom for confirmation by fluorescent antibody technique. This study reports a case of canine rabies in a vaccinated dog that poses a great public health risks. Differential diagnosis included foreign body lodgement in buccal cavity, tetanus, rabies, and helminthosis.

\section{Materials and Method}

Physical examination carried out on the carcass from head to tail. The head was cut with a sharp axe and wrapped up in a water-resistant polythene bag covered with cotton wool and an outer polythene bag before preservation in a freezer. Cold pack and chain were utilized in transporting the specimen from NVRI, Laboratory, Akure, to the Rabies Laboratory, NVRI, Vom. Craniotomy was performed to collect a portion of the hippocampus for fluorescent antibody technique as described [7]. Stated briefly, the brain smear was fixed in cold acetone, stained with light diagnostic antinucleocapsid monoclonal antibody, labeled with fluorescein isothiocyanate rabies fluorescent antibody assay DFA (monoclonal antibody FITC-conjugate), catalog no. 5100, reagent from Chemicon International Inc.; the stained smear was then observed for apple green fluorescence under the Leitz-Ortholux fluorescent microscope.

\section{Result and Discussion}

Mortality of the dog was recorded on the 9th day of quarantine, thus running 11 days course from the 1st day of sign manifestation. Physical examination that was carried out revealed emaciated carcass, locked jaw, or paralysed jaw muscle with protruded tongue. Diagnosis of rabies was made with the brain specimen that showed positive FAT and agreed with a confirmed case of rabies in a vaccinate by Seller's method [8]. This could be a result of vaccine failure due to a low immunogenic vaccine; infection by a more virulent Lyssavirus strain could also be a causal factor.

In this study, there was a major public health exposure risk considering the case as being vaccinated and other epidemiologic findings aforementioned. The dog as being vaccinated would reduce the suspicion of rabies. The observed signs were nonfurious which predisposes more people, particularly, the animal health care service provider, to bites by the dog and thus rabies.

Since the dog lived in unfenced apartment, her contact with other dogs especially stray male dogs for socialization is increased. This increases the transmission of rabies virus among contacted dogs and thus increased the zoonotic transmissibility. With increasing dog population as a result of human activities particularly in urban settings due to dog breeding as income source and security of live and properties, vaccination and immunogenic coverage should be improved to avert rabies. This increasing dog population with strays in every community could be a risk factor for increase incidence of dog bites that predisposes to rabies.

The role of laboratory testing and public health followup is reflected in parts by the number of costly postexposure prophylaxis potentially averted or discontinued [6]. The animal health care provider needs to be adequately protected with preexposure immunization.

Case determination should proceed beyond positive diagnosis of rabies especially for rabies involving vaccinated dogs. Rabies virus isolation and identification should be carried out as of necessity. This implied to positively updating the quality and quantity of virus bank for research and vaccinology. Most importantly, because vaccines in use are predominantly different types of foreign vaccines due to inadequate supply of the low and high egg passage (LEP and HEP) flurry rabies vaccines for dogs and cats, respectively, from NVRI, Vom, to meet the dog population increase.

One important point to note is that the appropriate authorities concerned do a contact tracing of the particular batch of the vaccine used. Starting with the case point or first point of disease reporting and vaccine administration point, also the dog owners should be traced followed by the determination of the vaccination and health status of their dogs. This intends to establish (1) point source case determination, (2) immunogenicity of the vaccine used, (3) identification and isolation of virus strain in circulation, and (4) possible vaccine induced outbreak.

This would invariably lead to appropriate feedback responses between clinician, veterinary authorities, that is, the state and NVRI, Vom, and Epidemiology Department, Federal Ministry of Agriculture and Rural Development, Abuja, thus ensuring adequate disease reporting and preventing accentuated zoonotic transmissibility of the rabies virus. Also, the epidemiology of this incident case would be adequately determined.

Conclusively, surveillance, in a closer coordination with local animal health authorities and community education [3], would strengthen the control of rabies in Nigeria.

\section{References}

[1] A. Z. Hassan, D. A. Y. Adawa, E. G. Emmanuel et al., "Behavioral conditions in Dogs. A review-behavioral disorders," The Veterinary Journal, vol. 30, no. 3, pp. 51-59, 2009.

[2] A. A. Dzikwi, J. U. Umoh, J. K. P. Kwaga, and A. A. Ahmad, "Serological surveillance for non-Rabies Lyssaviruses among apparently healthy dogs in Zaria," Nigerian Veterinary Journal, vol. 31, no. 3, pp. 214-218, 2010.

[3] R. Rimhanen-Finne, M. Kuusi, B. P. Quiambao et al., "Imported Human rabies, the Philipines and Finland," Emerging Infectious Diseases Journal, vol. 16, no. 8, pp. 1318-1319, 2010.

[4] G. Ahmed, Y. Dashe, C. I. Nwosuh et al., Rabies in puppies in Nigeria: An update 2006-2007. Book of Abstract, 45th Annual congress of NVMA, Owerri 2008, 56 Abstract, 2008.

[5] P. Crepin, L. Audry, Y. Rotivel, A. Gacoin, C. Caroff, and H. Bourhy, "Intravitam diagnosis of human rabies by PCR using 
saliva and cerebrospinal fluid," Journal of Clinical Microbiology, vol. 36, no. 4, pp. 1117-1121, 1998.

[6] E. Schelling, M. Bechir, M. A. Ahmed, K. Wyss, T. F. Randolph, and J. Zinsstag, "Human and animal vaccination delivery to remote nomadic families, Chad," Emerging Infectious Diseases, vol. 13, no. 3, pp. 373-378, 2007.

[7] Office International Des Epizooties. OIE manual on rabies, 2000.

[8] A. Ogbe, D. J. G. Mohammed, A. Obalisa, R. O. Grace, and N. J. Zwander, "Clinical evaluation of the circumstances of Dog bite in Man and the diagnosis of rabies in suspected Dogs in Vom, Plateau State," National Veterinary Research Institute Vom, seminar series 2001-2002, vol. 1, pp. 23-25, 2002. 

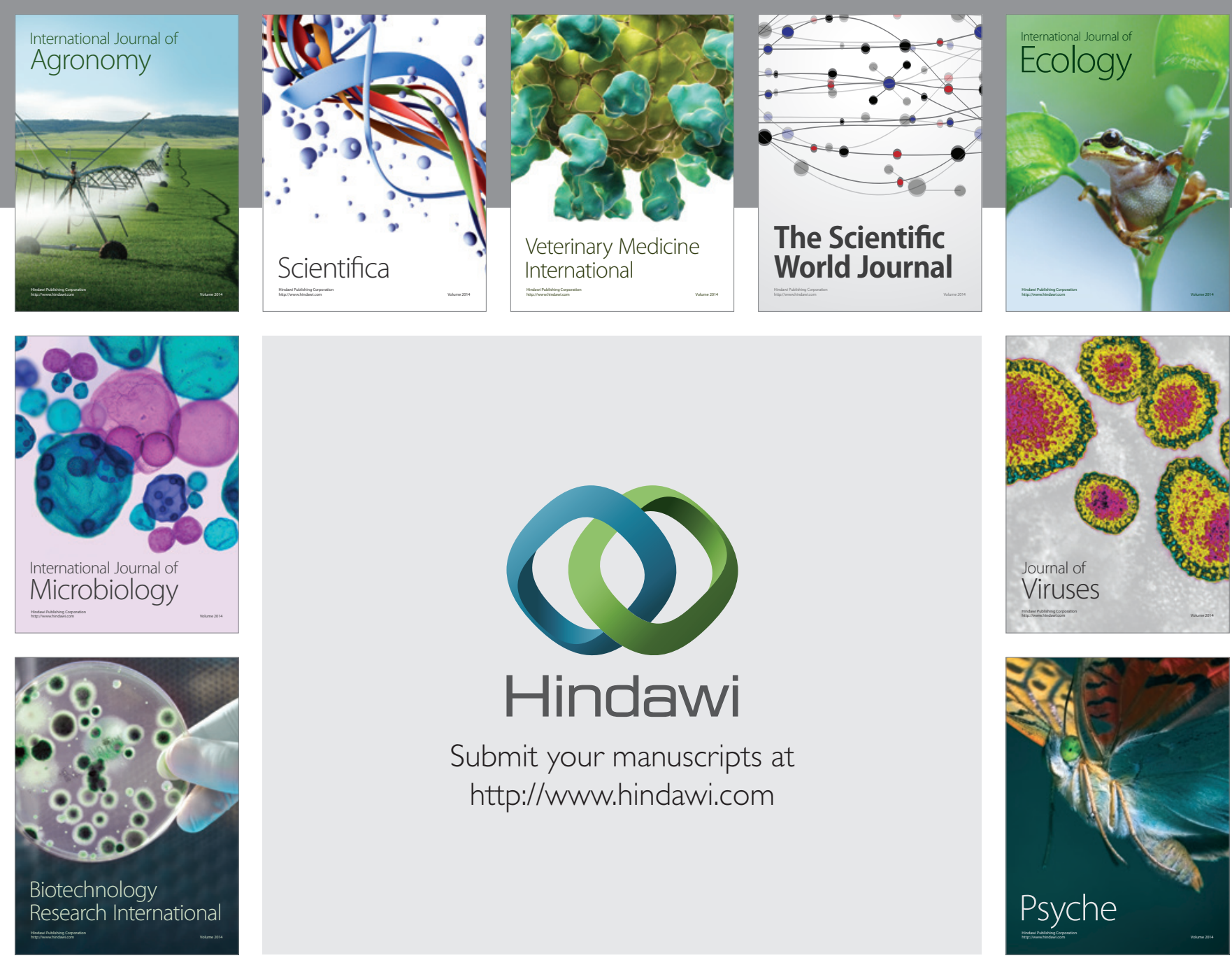

Submit your manuscripts at http://www.hindawi.com
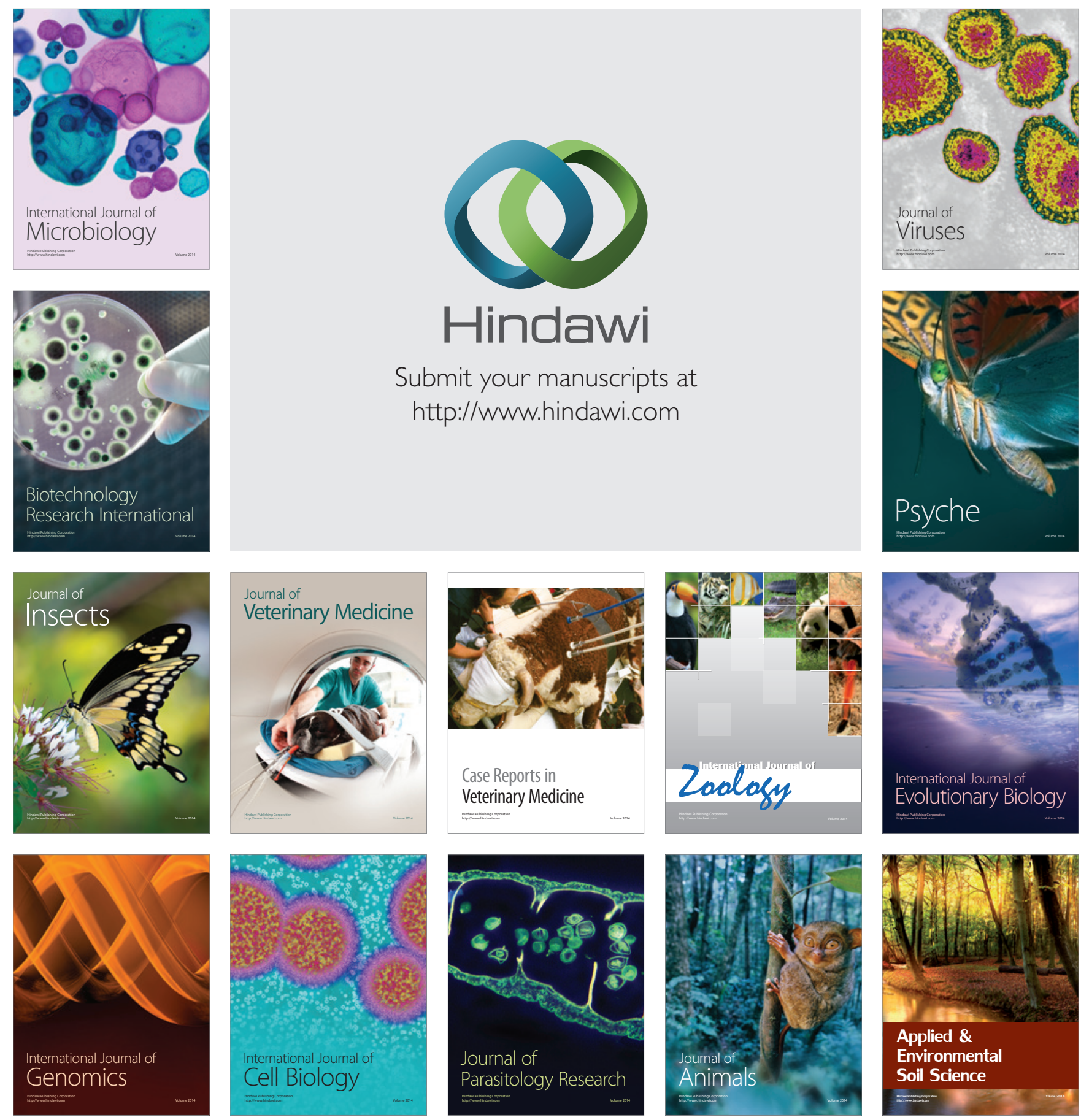